\title{
Chandra discovery of an X-ray jet and lobes in 3C 15
}

\author{
J. Kataoka ${ }^{1}$, J. P. Leahy ${ }^{2}$, P. G. Edwards ${ }^{3}$, M. Kino ${ }^{4,5}$, F. Takahara ${ }^{5}$, \\ Y. Serino ${ }^{1}$, N. Kawai ${ }^{1}$, and A. R. Martel ${ }^{6}$
}

1 Tokyo Institute of Technology, Meguro-ku, Tokyo, Japan

2 University of Manchester, Jodrell Bank Observatory, Macclesfield, Cheshire SK11 9DL, UK

3 Institute of Space and Astronautical Science, Sagamihara, Kanagawa, Japan

${ }^{4}$ Earth and Planetary Science, University of Tokyo, Japan

5 Department of Earth and Space Science, Osaka University, Osaka, Japan

${ }^{6}$ Space Telescope Science Institute, 3700 San Martin Drive, Baltimore, MD 21218, USA

Received 6 June 2003 / Accepted 13 August 2003

\begin{abstract}
We report the Chandra detection of an X-ray jet in 3C 15. The peak of the X-ray emission in the jet is $4.1^{\prime \prime}$ (a projected distance of $5.1 \mathrm{kpc}$ ) from the nucleus, and coincident with a component previously identified in the radio and optical jets. We construct the spectral energy distribution (SED) for this component, optical knot C, and find that X-ray flux is well below the extrapolation of the radio-to-optical continuum. We examine four models for the X-ray jet emission: (I) weak synchrotron cooling in equipartition, (II) moderate synchrotron cooling in equipartition, (III) weak synchrotron plus synchrotron self-Compton (SSC) cooling, and (IV) moderate synchrotron plus SSC cooling. Given weak evidence for a concave feature in the X-ray spectrum, we argue that case (II) can most reasonably explain the overall emission from knot C. Case (III) is also possible, but requires a large departure from equipartition and for the jet power to be comparable to that of the brightest quasars. In all models, (I)-(IV), electrons must be accelerated up to $\gamma_{\max } \gtrsim 10^{7}$, suggesting that re-acceleration is necessary in knot $\mathrm{C}$ of the $3 \mathrm{C} 15$ jet. Diffuse X-ray emission has also been detected, distributed widely over the full extent $(63 \mathrm{kpc} \times 25 \mathrm{kpc})$ of the radio lobes. The $\mathrm{X}$-ray spectrum of the diffuse emission is described by a two-component model, consisting of soft thermal plasma emission from the host galaxy halo and a hard nonthermal power-law component. The hard component can be ascribed to the inverse Comptonization of cosmic microwave background (CMB) photons by the synchrotron emitting electrons in the radio lobes. We compare the total energy contained in the lobes with the jet power estimated from knot $\mathrm{C}$, and discuss the energetic link between the jet and the lobes. We argue that the fueling time $\left(t_{\text {fuel }}\right)$ and the source age $\left(t_{\text {src }}\right)$ are comparable for case (II), whereas $t_{\text {fuel }} \ll t_{\text {src }}$ is likely for case (III). The latter may imply that the jet has a very small filling factor, $\sim 10^{-3}$. We consider the pressure balance between the thermal galaxy halo and non-thermal relativistic electrons in the radio lobes. Finally, we show that the $\mathrm{X}$-ray emission from the nucleus is not adequately fitted by a simple absorbed power-law model, but needs an additional power-law with heavy absorption $\left(N_{\mathrm{H}} \simeq 10^{22-23} \mathrm{~cm}^{-2}\right)$ intrinsic to the source. Such a high column density is consistent with the presence of a dense, dusty torus which obscures the quasar nucleus.
\end{abstract}

Key words. galaxies: active - galaxies: individual: 3C 15 - galaxies: jets - X-rays: galaxies

\section{Introduction}

Jets are among the most exciting (but also among the least understood) cosmic phenomena, being highly efficient particle accelerators that generate relativistic electron distributions. This extreme jet power is most likely generated near a super-massive black hole, probably via the conversion of gravitational energy from accreting matter. The interest in observations of jets at shorter wavelengths is related to the ability to (1) probe the sites of high energy particle acceleration and help determine their physical parameters, and (2) trace the energy transport from the nucleus to the outer hot spots/radio lobes. However, observations of jets with high spatial resolution $\left(\leq 1^{\prime \prime}\right)$ have

Send offprint requests to: J. Kataoka,

e-mail: kataoka@hp.phys.titech.ac.jp until recently only been possible at lower frequencies, i.e., at radio and optical wavelengths.

The excellent spatial resolution of the Chandra X-Ray Observatory has now resolved the X-ray spatial structure along the jets of more than 20 radio galaxies (e.g., Harris \& Krawczynski 2002, and references therein). The broadband spectral energy distributions (SEDs) of knots and hotspots show great variety between the radio and X-ray energy bands. In most cases, the X-ray spectra are much harder than expected from a simple extrapolation of the optical fluxes. This suggests that both the radio and optical photons are due to the same non-thermal synchrotron radiation, whereas X-ray photons are produced via Compton scattering of either synchrotron photons (SSC; e.g., Wilson et al. 2001 for the hotspot of Pictor A) and/or cosmic microwave background photons 
(EC/CMB; e.g., Sambruna et al. 2001 for the knots in 3C 273). In other cases, the X-ray-optical-radio spectrum is consistent with a single smoothly broken power-law spectrum, suggesting that the broad band emission is entirely due to non-thermal synchrotron radiation (e.g., Wilson \& Yang 2002 for the jet of M 87, and Hardcastle et al. 2001 for jet in 3C 66B).

The X-ray detections of jets and hotspots have a great impact on the determination of their physical parameters, as well as the maximum energy of accelerated electrons (see (1) above). Once the source of seed photons is identified, a comparison of the synchrotron (radio to X-ray) and inverse Compton (SSC or EC/CMB; X-ray) fluxes allows us to independently determine the energy densities of the relativistic electrons and the magnetic field, $u_{\mathrm{e}}$ and $u_{B}$ respectively. This approach has been applied to X-ray observations of radio lobes with $A S C A$ (Kaneda et al. 1995; Tashiro et al. 2001), in which the X-ray emission extends over arcmin scale sizes. By analyzing X-ray data of more than 10 radio lobes, Isobe (2002) found that lobes are generally "particle dominated", in the sense that $u_{\mathrm{e}} \geq u_{B}$. Recent Chandra detections of knots and hotspots have resulted in this idea being extended to more compact regions in jets with $\sim$ arcsec spatial extents. In some objects, the X-ray fluxes of knots and hotspots turned out to be much brighter than that expected from equipartition between particles and fields (e.g., Hardcastle et al. 2002; Kataoka et al. 2003; Georganopoulos \& Kazanas 2003).

Comparison of the powers in jets and lobes provides an important clue to the formation of astrophysical jets and the evolution of radio galaxies (see (2) above). This is because lobes are undoubtedly fueled by jets over a long time, possibly throughout their lifetime. Assuming the total power of the jet $\left(L_{\mathrm{E}}\right)$ and the total energy supplied in the radio lobes $\left(E_{\text {lobe }}\right)$, the "fueling time" of the system may be defined as $t_{\text {fuel }} \sim E_{\text {lobe }} / L_{\mathrm{E}}$. Meanwhile, the "source age" can be defined as $t_{\mathrm{src}} \sim D / v_{\exp }$, where the $D$ is the spatial extent and $v_{\text {exp }}$ is the expansion speed of the radio lobes. Observationally, symmetry arguments show that the main-axis expansion of FR II lobes is sub-relativistic $\left(v_{\exp } \leq 0.1 c\right.$; e.g., Scheuer 1995), and since the lobes are not spherical the limit to transverse expansion is several times lower. In fact, lobes are surrounded by hot plasma which will prevent them expanding freely, either by thermal or by rampressure confinement (e.g., Leahy \& Gizani 2001). We expect $t_{\text {fuel }} \sim t_{\text {src }}$ if the jet power is injected into the radio lobes in a stationary, constant manner. A large departure from this equality may imply that either the injection of power by the jet is not uniform, and/or that there are significant contributions from particles which we cannot observe. Comparison of $t_{\text {fuel }}$ and $t_{\text {src }}$ provides important information for understanding the jet-lobe connection.

3C 15 is an unusual radio source with an optical jet which has been imaged with the Hubble S pace Telescope (HST). The $H S T$ observations found no bright, unresolved nucleus in the core of the galaxy, in contrast to other galaxies hosting optical jets (Martel et al. 1998). The radio structure of 3C 15 is intermediate between Fanaroff-Riley (FR) classes I and II, although its luminosity is above that of a number of sources showing classical FR II structure (Leahy et al. 1997). The optical morphology of the jet matches the features of the radio jet very well, suggesting that the optical emission is strongly dominated by synchrotron radiation. In this paper, we report the Chandra discovery of X-ray emission from the knots, lobes and nucleus of $3 \mathrm{C} 15$. By combining the data from radio to X-rays, we derive the power of the jet and the lobes separately for extensive study. Throughout this paper, we adopt $H_{0}=75 \mathrm{~km} \mathrm{~s}^{-1} \mathrm{Mpc}^{-1}$ and $q_{0}=0.5$, so that $1^{\prime \prime}$ corresponds to $1.25 \mathrm{kpc}$ at the redshift of $3 \mathrm{C} 15, z=0.073$.

\section{Observations}

\subsection{Summary of previous radio observations}

$3 \mathrm{C} 15$ has a bright northern jet containing four prominent knots, with a much weaker counter-jet on the southern side of the nucleus. VLA observations at $8.3 \mathrm{GHz}$ were made by Leahy et al. (1997), and we use these data in this paper, with an 0.34 arcsec resolution image shown as contour levels in Fig. 1 (upper right). The nucleus and jets are surrounded by lobe emission, which extends over 50 arcsec (a projected distance of $63 \mathrm{kpc}$ ). The surface brightness profile of $3 \mathrm{C} 15$ projected onto the major axis of the radio lobes is shown in Fig. 2. Leahy et al. (1997) labeled the four radio knots A, B, C and D, however, the optical observations described in the next section resolved the radio knot $\mathrm{A}$ into two optical knots. In this paper we follow the optical scheme of labeling the knots A and B (corresponding to radio knot $\mathrm{A}$ ) and $\mathrm{C}$ (corresponding to radio knot $\mathrm{B}$ ). The jet-counter-jet flux density ratio is $50 \pm 10$ (Leahy et al. 1997). The VLA images suggests the source does not lie in the plane of the sky, in which case the jet asymmetry may partially be due to relativistic beaming. Assuming a spectral index, $\alpha_{\mathrm{R}}$, of 0.6 (where $S \propto v^{-\alpha_{\mathrm{R}}}$ ), the flux asymmetry implies $\beta \cos \theta=0.635 \pm 0.025$, hence $\theta \simeq 45-50^{\circ}$ for $\beta \geq 0.9$. This corresponds to a Doppler beaming factor in the range $0.40 \leq \delta \leq 1.2$.

\subsection{Summary of previous optical observations}

The optical counterpart of the radio jet in 3C 15 was found in $H S T$ observations by Martel et al. (1998), and we use these $H S T$ data in this paper. The optical morphology of the jet closely matches the features of the radio jet, suggesting that the optical emission is strongly dominated by synchrotron radiation (Fig. 5 of Martel et al. 1998). Three prominent knots in the jet were detected at a PA of $-30^{\circ}$. Thanks to the excellent image resolution of the HST $\left(0.1^{\prime \prime}\right)$, the size of each knot has been accurately measured. The innermost knot, knot $\mathrm{A}$, is broad and diffuse. It extends from $2.6 \mathrm{kpc}\left(2.1^{\prime \prime}\right)$ to $3.5 \mathrm{kpc}\left(2.8^{\prime \prime}\right)$ from the nucleus. Knots $\mathrm{B}$ and $\mathrm{C}$ are comparatively compact and well defined. The FWHMs of knot B and C are both $\sim 0.5 \mathrm{kpc}$. The outermost knots of the radio jet, defined as $\mathrm{C}$ and $\mathrm{D}$ by Leahy et al. (1997) are not detected in the optical image. Martel et al. (1998) compared the fluxes of the jet measured in the $V$ and $R$ bands with radio and soft $\mathrm{X}$-ray data. The low resolution radio data and the $\mathrm{X}$-ray observations made by ROSAT include flux contributions from both the jet and the nucleus of $3 \mathrm{C} 15$. A fit through the radio to optical data yields $\alpha_{\mathrm{ro}}=1.03 \pm 0.02$. When the flux measurement of only the northern jet (combined 

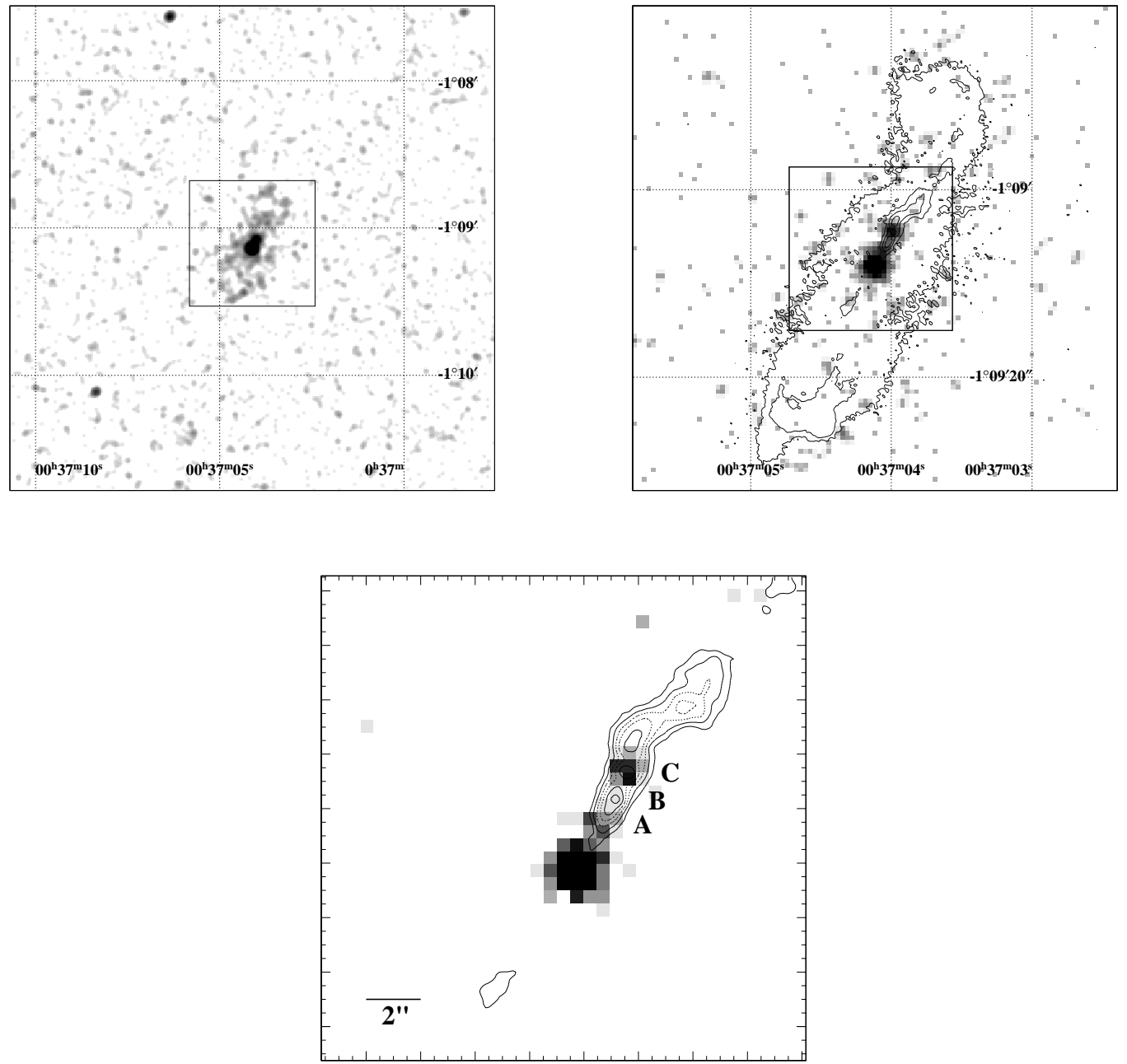

Fig. 1. Upper left: a $0.4-8 \mathrm{keV}$ X-ray image of 3C 15 (Chandra ACIS-S3) smoothed with a two-dimensional Gaussian of $\sigma=1.0$ arcsec. Upper right: radio and X-ray image of 3C 15 lobes and jet (expanded plot of the solid square in the left panel). The grey scale is the X-ray image smoothed with a $\sigma=0.3$ arcsec Gaussian. The VLA $8.3 \mathrm{GHz}$ image is adapted from Leahy et al. (1997). The contour levels are (0.02, $0.11,0.62,3.5,20) \mathrm{mJy}_{\text {beam }}^{-1}$ for a beam size of $0.34^{\prime \prime} \times 0.34^{\prime \prime}$. Bottom: expanded plot of the central jet region $\left(17.4^{\prime \prime} \times 17.4^{\prime \prime}\right)$ shown by the solid square in the upper right panel). The image is smoothed with a $\sigma=0.3 \operatorname{arcsec}$ Gaussian. A, B, and $\mathrm{C}$ denote the jet knots as defined in Martel et al. (1998). The VLA contour levels are $(0.2,0.56,1.6,4.3,12) \mathrm{mJy}^{-1}$ beam ${ }^{-1}$ for a beam size of $0.34^{\prime \prime} \times 0.34^{\prime \prime}$.

knots $\mathrm{A}, \mathrm{B}$, and $\mathrm{C}$ ) is considered, a radio-optical spectral index of $0.95 \pm 0.01$ is obtained.

\subsection{X-ray observation and data reduction}

3C 15 was observed by the Chandra X-ray Observatory on 2000 November 6 in a guaranteed time observation (sequence number 700368, obs. ID 2178) using the Advanced CCD Imaging Spectrometer (ACIS-S) spectroscopic array. The nucleus of $3 \mathrm{C} 15$ was centered $20^{\prime \prime}$ in the $-Y$ direction from the location of the best focus on chip S3 (nominal position of the ACIS-S3 chip). All of the regions of radio emission from $3 \mathrm{C} 15$ were imaged on S3. The total good time interval was $28.2 \mathrm{ksec}$, taken with the default frame time of $3.2 \mathrm{~s}$. We have analyzed archival data on 3C15 provided by HEASARC Browse (http://heasarc.gsfc.nasa.gov/dp-perl/W3Browse/ Browse.p1). The raw level-1 data were reprocessed using the latest version (CIAO 2.2.1) of the CXCDS software and version 11.1.0 of XSPEC. We generated a clean data set by selecting the standard grades $(0,2,3,4$ and 6$)$ and the energy band $0.4-8 \mathrm{keV}$.

\section{Results}

\subsection{X-ray image}

The X-ray image, produced by smoothing the raw Chandra image in the energy range of $0.4-8 \mathrm{keV}$ with a Gaussian of width $1.0^{\prime \prime}$ is shown in Fig. 1 (upper left). We find faint $\mathrm{X}$-ray emission surrounding the nucleus, extended in the north-west-south-east direction. Comparing this to the $8.3 \mathrm{GHz}$ VLA image in Leahy et al. (1997), the extended X-ray emission appears to be correlated with the extended radio lobes. An expanded plot of the solid square in the left figure is shown in the right panel, where the radio contours are superposed on the X-ray image (grey scale) smoothed with a $\sigma=0.3 \operatorname{arcsec}$ Gaussian. X-ray emission can be clearly seen from the bright central nucleus and the northern jet. 


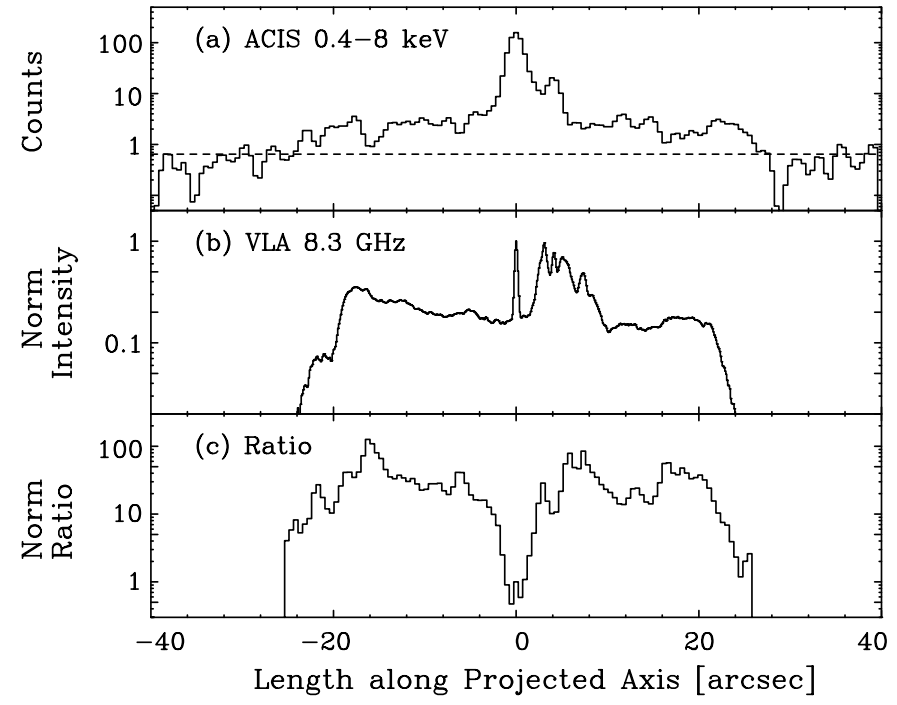

Fig. 2. Log profiles along the major axis of the lobes of $3 \mathrm{C} 15$. a) The $\mathrm{X}$-rays in the $0.4-8 \mathrm{keV}$ range, b) the radio emission at $8.3 \mathrm{GHz}$ (normalized to the nuclear radio flux), and c) the ratio between the radio and X-ray profiles ( $S_{\text {radio }} / S_{\mathrm{X}}$; normalized to central nucleus).

An expanded plot of the central jet region $\left(17.4^{\prime \prime} \times 17.4^{\prime \prime}\right)$ is shown in the bottom panel. The nucleus and jet knots are detected at the $22.9 \sigma$ (527 counts) and $7.7 \sigma$ (60 counts for knot C: see below) level, respectively. The number of net X-ray photons associated with the radio lobes is 246 photons, which includes a background of 73 photons in the $0.4-8 \mathrm{keV}$ range (11 $\sigma$ level), where we have excluded pixels within $1.9^{\prime \prime}$ of the possible point sources, including knot $\mathrm{C}$, knot $\mathrm{A}^{\prime}$ and the nucleus (see below).

In order to examine the spatial correlation between the radio and X-ray emission, we projected the raw ACIS image onto the major axis of the radio lobes. Here the strip integration is restricted to a width of $20^{\prime \prime}$ transverse to the major axis. The X-ray logarithmic surface brightness profile is shown in Fig. 2a, together with the radio profile in Fig. 2b. The X-ray profile has been smoothed with a Gaussian of width $1^{\prime \prime}$. No background subtraction has been performed in the X-ray image, hence the profile contains an average background of 0.64 counts/bin in the $0.4-8.0 \mathrm{keV}$ band (given as the dashed line in Fig. 2a). Although the X-ray photon statistics are limited, the X-ray and radio profiles clearly show similar spatial extents but different structures. The X-ray profile exhibits a "center-filled" morphology with a relatively uniform surface brightness (except for the nucleus region), while the northern jet and southern rim are bright in the radio image. These differences can also be seen in the bottom panel, where the surface brightness ratio ( $S_{\text {radio }} / S_{\mathrm{X}}$; normalized to the nucleus) is a minimum at the center, and gradually increases at larger distances.

The expanded profile of the X-ray jet is shown in Fig. 3, together with the radio (VLA $8.3 \mathrm{GHz}$ ) and optical $(H S T)$ jet profiles. Note that the strong background light of host galaxy is subtracted in the optical profile, resulting in only the jet knots being visible (see also Sect. 4.3). The X-ray image resolution is poorer than that of the optical and radio, but it is clear that most of the X-ray jet emission originates from the knot $\mathrm{C}$ of

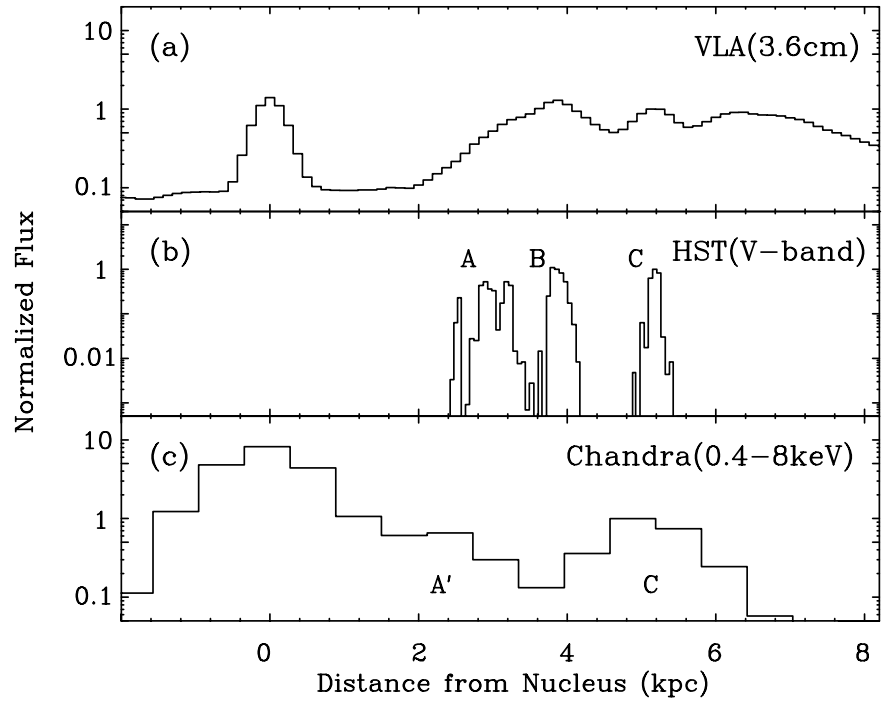

Fig. 3. $\log$ profiles along the major axis of the jet of $3 \mathrm{C} 15$, a) the radio emission at $8.3 \mathrm{GHz}, \mathbf{b})$ the $V$ band ( $H S T$; adapted from Fig. 3 in Martel et al. 1998), and c) the X-rays in the $0.4-8 \mathrm{keV}$ range. All jet profiles are normalized to the knot $\mathrm{C}$ flux. The underlying galactic light, and hence bright nuclear emission, are subtracted in the optical profile b).

Martel et al. (1998). The total extent (FWHM) of the X-ray nucleus and knot $\mathrm{C}$ are both $\sim 1^{\prime \prime}$, consistent with the the broadening of the Chandra PSF ( $\sim 0.5^{\prime \prime}$ half-energy radius). There is also a suggestion of faint X-ray emission from the northern jet intermediate between the nucleus and knot $\mathrm{C}$ (denoted $\mathrm{A}^{\prime}$ in Fig. 3c), although this is heavily contaminated by the bright nuclear emission. This feature is located $1.8 \pm 0.1^{\prime \prime}$ from the nucleus, and contains $45 \pm 12$ photons. This position is approximately coincident with the innermost knot detected by $H S T$ (knot A; see Sect. 2.2), however the offset of (0.2-0.9) $\pm 0.1^{\prime \prime}$ between the X-ray and optical peaks, with the X-ray peak being closer to the nucleus, seems to be significant. Future observations with improved photon statistics and image resolution are necessary to confirm this.

\subsection{X-ray spectrum of knot $C$}

We extracted the X-ray photons from knot $\mathrm{C}$ within a circular region of $1.6^{\prime \prime}$ radius, to reduce the contamination of photons from knot $\mathrm{A}^{\prime}$ (to less than $\sim 10 \%$ ). Approximately $97 \%$ of the counts from a point source are collected within a circular region of this size. Background subtraction was performed, where the background counts were accumulated at the same off-nuclear distance. Figure 4 shows the backgroundsubtracted ACIS spectrum of knot C. We first assumed a powerlaw function absorbed by Galactic $N_{\mathrm{H}}$ only (fixed to $3.03 \times$ $10^{20} \mathrm{~cm}^{2}$ : Stark et al. 1992). The best fit X-ray photon index was $\Gamma_{\text {jet }}=1.71_{-0.36}^{+0.44}$, and the corresponding $0.5-5 \mathrm{keV}$ flux was $\left(8.38_{-1.85}^{+1.83}\right) \times 10^{-15} \mathrm{erg} \mathrm{cm}^{-2} \mathrm{~s}^{-1}$. The reduced $\chi^{2}$ is 3.1 for 2 degrees of freedom, which corresponds to $P\left(\chi^{2}\right)=5 \%$. Although the photon spectrum can formally be fitted by a simple power-law function, in the sense that $P\left(\chi^{2}\right) \geq 5 \%$, it is apparent that the model does not provide the best possible fit to 
Table 1. Best-fit spectral parameters for knot $\mathrm{C}$ of $3 \mathrm{C} 15$.

\begin{tabular}{lcllll}
\hline \hline Model & ${\text { Photon Index } 1^{a}}$ & Photon Index $2^{b}$ & ${\text { Break } E(\mathrm{keV})^{c}}$ & $0.5-5 \quad \mathrm{keV} \mathrm{flux}$ & red. $\chi^{2}$ (d.o.f.) \\
\hline PL & $1.71_{-0.36}^{+0.44}$ & $\ldots$ & $\ldots$ & $\left(8.38_{-1.85}^{+1.83}\right) \times 10^{-15}$ & $3.1(2)$ \\
Broken PL & 3.0 (fixed) & 1.5 (fixed) & $0.90 \pm 0.18$ & $\left(8.73_{-2.02}^{+1.85}\right) \times 10^{-15}$ & $0.98(2)$ \\
\hline
\end{tabular}

${ }^{a}$ The best-fit power-law photon index assuming a Galactic $N_{\mathrm{H}}$ of $3.03 \times 10^{20} \mathrm{~cm}^{-2}$.

${ }^{b}$ The best-fit power-law photon index above the break for a broken power-law model.

${ }^{c}$ The best-fit break energy for a broken power-law model.

${ }^{d}$ In units of erg $\mathrm{cm}^{-2} \mathrm{~s}^{-1}$ (absorption corrected).

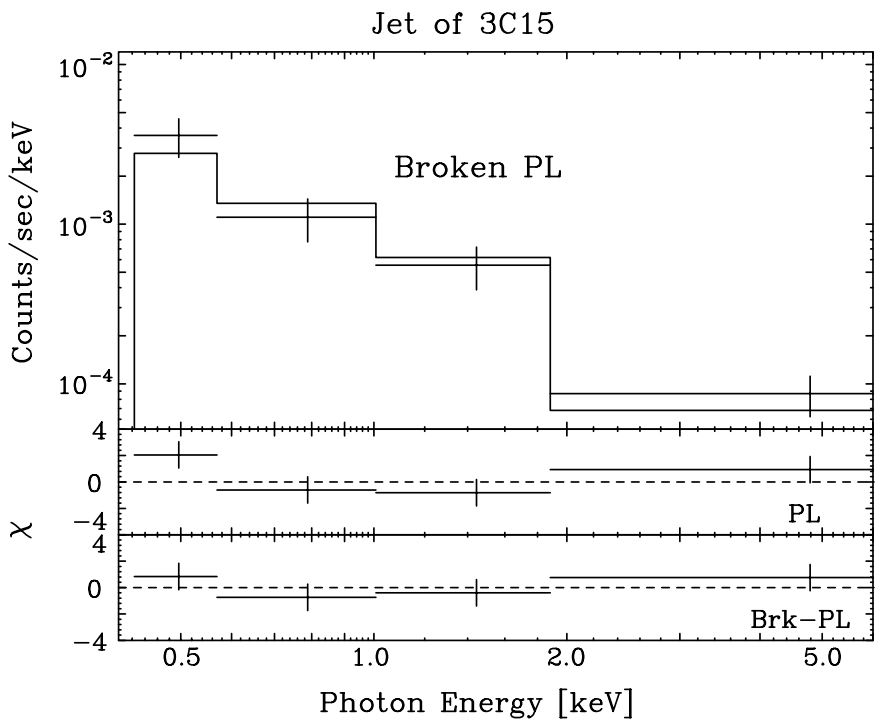

Fig. 4. Background-subtracted ACIS spectrum of knot $\mathrm{C}$ fitted by a broken power-law function. The middle panel shows the residuals to the power-law fit with a differential photon index of $\Gamma=1.7$. A concave feature is apparent in the residuals. The bottom panel shows the residuals to the best-fit broken power-law model fixed at $\Gamma_{1}=3.0$ and $\Gamma_{2}=1.5$.

the data; the observed X-ray spectrum show a concave feature with a break around $1 \mathrm{keV}$ (Fig. 4). Spectral fits with a broken power-law model improve the goodness of the fit significantly, as summarized in Table 1. Note that, below this break energy, the photon spectrum shows a steep power-law index of $\Gamma_{\mathrm{L}} \sim 3.0$, which flattens appreciably to $\Gamma_{\mathrm{H}} \sim 1.5$ above the break. The $0.5-5 \mathrm{keV}$ flux was estimated to be $\left(8.73_{-2.02}^{+1.85}\right) \times$ $10^{-15} \mathrm{erg} \mathrm{cm}^{-2} \mathrm{~s}^{-1}$.

\subsection{X-ray spectrum of the lobes}

To examine the diffuse X-ray emission from the radio lobes, we accumulated an ACIS spectrum within an elliptical region containing the whole radio structure. An ellipse of $60^{\prime \prime} \times 30^{\prime \prime}$ centered on the nucleus was selected, excluding regions around the jet and the nucleus (see above) and those within $2^{\prime \prime}$ of a possible point source. We accumulated the background spectrum over a circular region of 1 arcmin radius centered on the nucleus, excluding the region of the lobes. Figure 4 shows the background-subtracted ACIS spectrum of the lobes of 3C 15 in the $0.5-5 \mathrm{keV}$ range. The X-ray spectrum could not be fitted well by either a simple Raymond-Smith (RS) model or

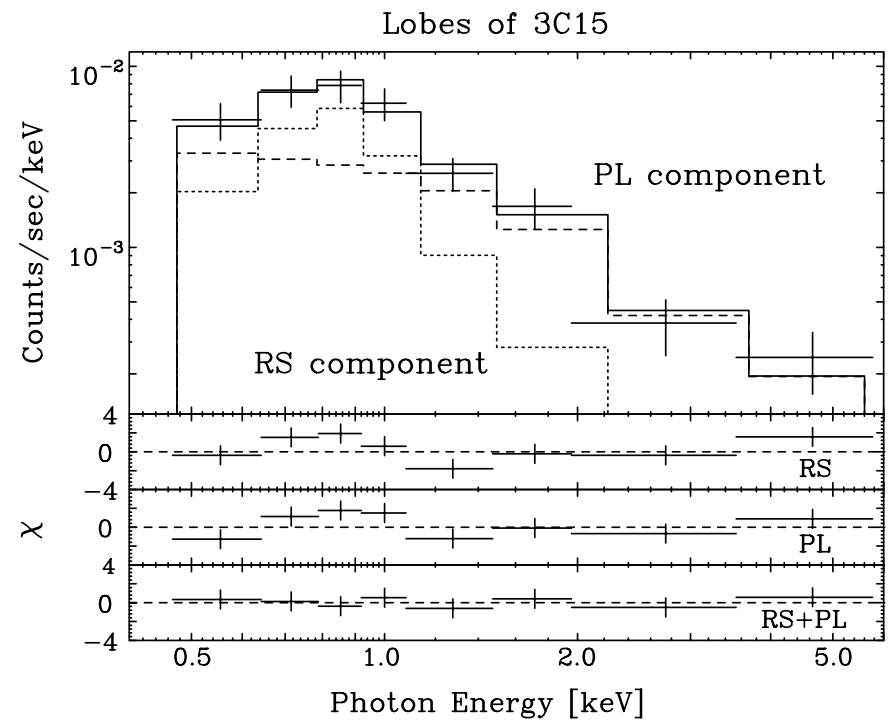

Fig. 5. Background-subtracted ACIS spectrum of the lobes of 3C 15 . The dotted and dashed histograms represent the best-fit soft RS and hard PL components, respectively. The solid line shows their sum. The second panel shows the residuals to the Raymond-Smith fit with a temperature of $k T=2.9 \mathrm{keV}$. The third panel shows the residuals to the power-law fit with differential photon index $\Gamma=1.9$. The bottom panel shows the residuals to the best-fit Raymond-Smith plus powerlaw model.

a power-law (PL) function. The $\chi^{2}$ were 2.1 and 1.8 for 6 degrees of freedom, respectively $\left(P\left(\chi^{2}\right)=0.05,0.09\right)$, with a significant excess around $0.7-1.0 \mathrm{keV}$. We thus fitted the data by a single PL plus RS model with the abundance fixed at 0.4 solar. The obtained parameters are summarized in Table 2, and results of fits by PL+RS model is shown in Fig. 5. The total X-ray flux of the radio lobes is calculated to be $\left(3.18_{-0.61}^{+0.75}\right) \times 10^{-14} \mathrm{erg} \mathrm{cm}^{-2} \mathrm{~s}^{-1}$ in the $0.5-5 \mathrm{keV}$ energy range. The X-ray luminosity of the thermal component fitted to $\mathrm{RS}$ model is $L_{\mathrm{RS}}=\left(4.51_{-1.91}^{+2.99}\right) \times 10^{40} \mathrm{erg} \mathrm{s}^{-1}$, whereas $L_{\mathrm{PL}}=$ $\left(1.25_{-0.46}^{+0.42}\right) \times 10^{41} \mathrm{erg} \mathrm{s}^{-1}$ for the non-thermal PL component.

\subsection{X-ray spectrum of the nucleus}

$\mathrm{X}$-ray photons from the nucleus were extracted from a circular region of $1.9^{\prime \prime}$ radius (within which approximately $98 \%$ of counts from a point source would be collected). Contamination of photons from knot $\mathrm{A}^{\prime}$ is less than $1 \%$. The background photons were accumulated over the lobe region. Figure 6 shows the background-subtracted ACIS spectrum of the 
Table 2. Best-fit spectral parameters for the lobes of $3 \mathrm{C} 15$.

\begin{tabular}{lcllllll}
\hline \hline Model & $k T(\mathrm{keV})^{a}$ & Abundance $^{b}$ & RS flux $(0.5-5 \quad \mathrm{keV})^{c}$ & Photon $^{\text {Index }}{ }^{d}$ & PL flux $(0.5-5$ & $\mathrm{keV})^{e}$ & red. $\chi^{2}($ d.o.f.) \\
\hline RS & $2.9_{-0.9}^{+1.6}$ & 0.4 (fixed) & $(2.68 \pm 0.23) \times 10^{-14}$ & $\ldots$ & $\ldots$ & \\
PL & $\ldots$ & $\ldots$ & $\ldots$ & $1.85_{-0.16}^{+0.17}$ & $\left(3.07_{-0.34}^{+0.35}\right) \times 10^{-14}$ & $1.1(6)$ \\
RS+PL & $0.85_{-0.08}^{+0.14}$ & 0.4 (fixed) & $\left(8.70_{-3.73}^{+5.80}\right) \times 10^{-15}$ & $1.33_{-0.43}^{+0.35}$ & $(2.31 \pm 0.48) \times 10^{-14}$ & $0.41(4)$ \\
\hline
\end{tabular}

${ }^{a}$ The best-fit plasma temperature for the Raymond-Smith model, assuming a Galactic $N_{\mathrm{H}}$ of $3.03 \times 10^{20} \mathrm{~cm}^{-2}$.

${ }^{b}$ Metal abundance for the Raymond-Smith model.

${ }^{c}$ Flux of the Raymond-Smith model in units of $\mathrm{erg}_{\mathrm{cm}}{ }^{-2} \mathrm{~s}^{-1}$ (absorption corrected).

${ }^{d}$ The best-fit power-law photon index.

${ }^{e}$ Flux of the power-law model in units of $\mathrm{erg} \mathrm{cm}^{-2} \mathrm{~s}^{-1}$ (absorption corrected).

Table 3. Best-fit spectral parameters for the nucleus of 3C 15 .

\begin{tabular}{|c|c|c|c|c|c|c|}
\hline Model & Photon Index $1^{a}$ & $0.5-5 \mathrm{keV}$ flux $1^{b}$ & Absorbed $N_{\mathrm{H}}^{c}$ & Photon Index $2^{d}$ & $0.5-5 \mathrm{keV} \mathrm{flux} 2^{e}$ & red. $\chi^{2}$ (d.o.f.) \\
\hline PL & $0.45 \pm 0.09$ & $(1.37 \pm 0.07) \times 10^{-13}$ & $\ldots$ & $\ldots$ & $\ldots$ & $1.45(22)$ \\
\hline Dbl-PL & $1.28_{-0.17}^{+0.18}$ & $\left(6.81_{-1.00}^{+1.14}\right) \times 10^{-14}$ & $7.0 \times 10^{22}($ fixed $)$ & $1.92_{-0.46}^{+0.41}$ & $\left(4.47_{-1.61}^{+2.40}\right) \times 10^{-13}$ & $0.55(20)$ \\
\hline
\end{tabular}

${ }^{a}$ The best-fit power-law photon index for PL1.

${ }^{b} 0.5-5 \mathrm{keV}$ flux for PL1 in units of erg $\mathrm{cm}^{-2} \mathrm{~s}^{-1}$ (absorption corrected).

${ }^{c}$ Absorbed column density for PL2.

${ }^{d}$ The best fit power-law photon index for PL2.

${ }^{e} 0.5-5 \mathrm{keV}$ flux for PL2 in units of $\mathrm{erg}^{-2} \mathrm{~cm}^{-1}$ (absorption corrected).

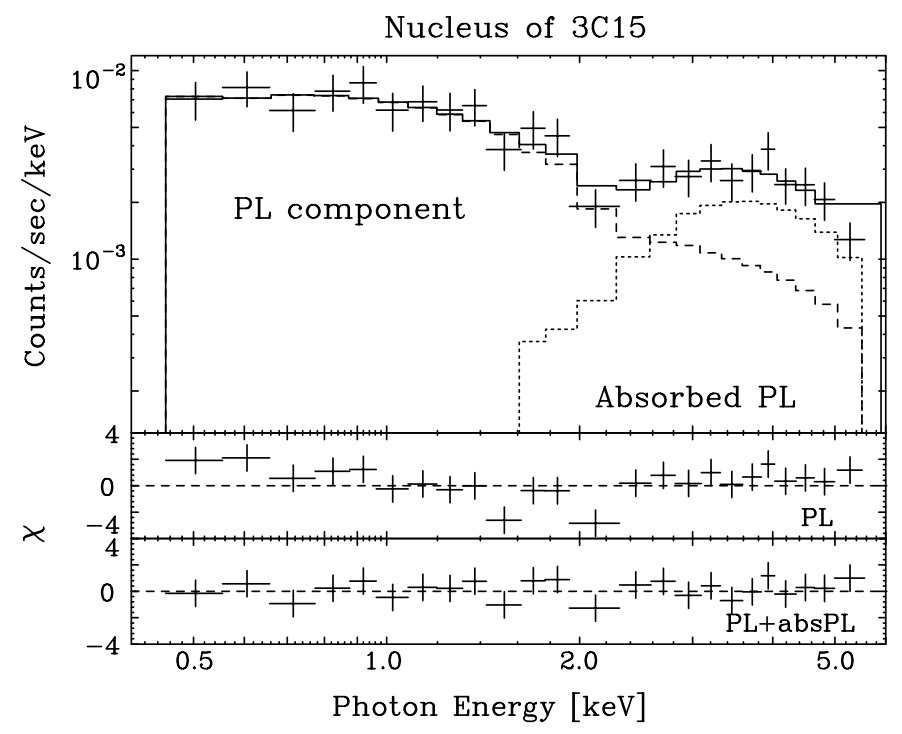

Fig. 6. Background-subtracted ACIS spectrum of the nucleus of 3C 15. The best-fit model, consisting of two power-law components, is shown with the histograms. The middle panel shows the residuals to the power-law fit with a differential photon index $\Gamma=0.5$. The bottom panel shows the residuals to the best-fit double power-law model.

nucleus. A high energy bump is seen in the spectrum, which cannot be fitted by a simple power-law function (red. $\chi^{2}=32.0$ for 22 degrees of freedom; $\left.P\left(\chi^{2}\right)=0.08\right)$. We therefore adopt a model consisting of two power-law functions, one of which is modified by Galactic absorption (PL1) with the other (PL2) having a heavily absorbed neutral hydrogen column density intrinsic to the source. A satisfactory fit was obtained if we assumed $N_{\mathrm{H}}=\left(7.3_{-3.9}^{+6.0}\right) \times 10^{22} \mathrm{~cm}^{-2}$ for PL2. In order to reduce the error in the power-law index for PL2, we thus fixed $N_{\mathrm{H}}$ to $7.0 \times 10^{22} \mathrm{~cm}^{-2}$ and refit the data. The best-fit parameters are summarized in Table 3 . The absorption corrected fluxes were $\left(6.81_{-1.00}^{+1.14}\right) \times 10^{-14} \mathrm{erg} \mathrm{cm}^{-2} \mathrm{~s}^{-1}$ for PL1, and $\left(4.47_{-1.61}^{+2.40}\right) \times$ $10^{-13} \mathrm{erg} \mathrm{cm}^{-2} \mathrm{~s}^{-1}$ for PL2, respectively. The corresponding X-ray luminosities are $L_{\mathrm{PL} 1}=\left(3.52_{-0.52}^{+0.59}\right) \times 10^{41} \mathrm{erg} \mathrm{s}^{-1}$, and $L_{\mathrm{PL} 2}=\left(2.31_{-0.83}^{+1.24}\right) \times 10^{42} \mathrm{erg} \mathrm{s}^{-1}$, respectively.

\section{Discussion}

\subsection{Emission mechanism of knot $C$}

As described in the previous sections, we have detected the X-ray counterpart of the radio-optical jet-knot in the radio galaxy $3 \mathrm{C} 15$. Figure 7 shows the SED from radio to X-ray energies of knot $\mathrm{C}$. Since only the integrated fluxes of the radio and optical knots (from A to $\mathrm{C}$ or D) are given in the literature, we calculated the radio and optical fluxes of knot $\mathrm{C}$ by direct integration from the radio and optical images. These are $35 \mathrm{mJy}$ for $8.3 \mathrm{GHz}$ and $6.5 \mu \mathrm{Jy}$ for $V$ band, respectively (see Leahy et al. 1997 and Martel et al. 1998 for the integrated jet fluxes). Figure 7 clearly indicates that the X-ray flux obtained with Chandra is well below the extrapolation from the radioto-optical continuum. A power-law fit $\left(S_{v} \propto v^{-\alpha}\right.$, where $S_{v}$ is the flux density) through the radio and optical points yields a spectral index of $\alpha_{\text {ro }}=0.9$, whereas a power-law fit through the optical and X-ray (at $1 \mathrm{keV}$ ) data yields a steeper spectral index of $\alpha_{\mathrm{ox}} \simeq 1.1-1.2$. This fact suggests that the synchrotron peak in the $v F_{v}$ plot must lie below the X-ray energy bands. However, the $\mathrm{X}$-ray emission process of knot $\mathrm{C}$ is currently uncertain due to the poor photon statistics (Fig. 4): in fact, we cannot conclude if the X-ray spectrum is "falling" or "rising" in the $v F_{v}$ SED plane. The former case would suggest that the $\mathrm{X}$-rays are produced by pure synchrotron emission, as for 

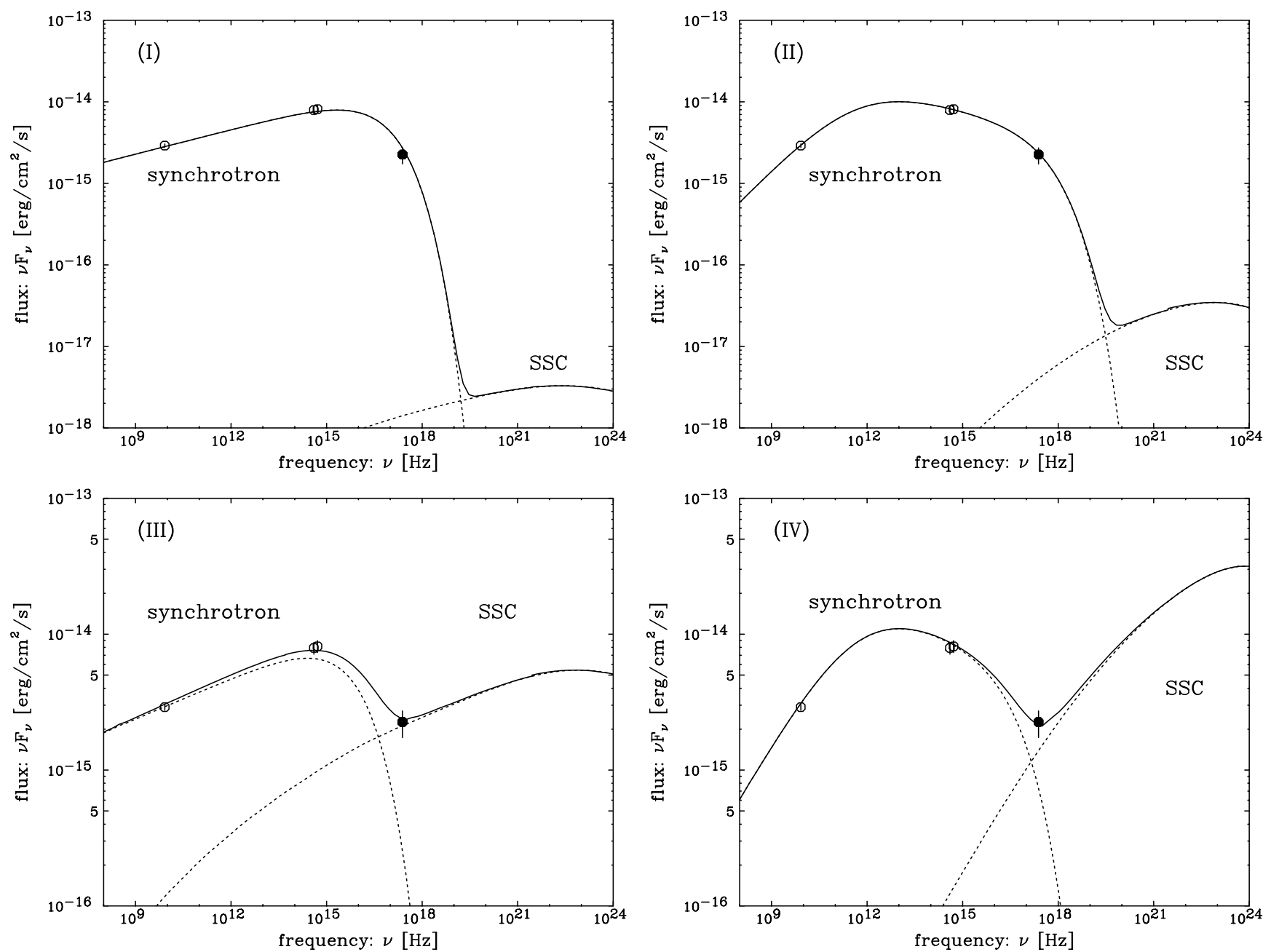

Fig. 7. SED of knot $\mathrm{C}$ of the $3 \mathrm{C} 15$ jet. The radio and optical fluxes are calculated by direct integration from the radio and optical images given in Leahy et al. (1997) and Martel et al. (1998). The X-ray data are from the Chandra observations (this work; $1 \sigma$ error at $1 \mathrm{keV}$ flux). The four models considered in the text are plotted: (I) weak synchrotron cooling for $u_{\mathrm{e}}=u_{B}$, (II) moderate synchrotron cooling for $u_{\mathrm{e}}=u_{B}$, (III) weak synchrotron plus SSC cooling, and (IV) moderate synchrotron plus SSC cooling. The fitting parameters are summarized in Table 4.

the radio-to-optical bands, whereas the latter would imply that the X-rays are the low end of the hard inverse Compton (IC) emission. In the following, we thus consider both these scenarios in discussing the jet parameters.

A power-law energy injection of electrons up to a certain maximum energy $\gamma_{\max }$ (as expected from shock acceleration) into the radiating region should yield a steady-state electron distribution approximated by

$N(\gamma)=N_{0} \gamma^{-\mathrm{s}}\left(1+\frac{\gamma}{\gamma_{\text {brk }}}\right)^{-1} \exp \left(-\frac{\gamma}{\gamma_{\text {max }}}\right)$,

where $s$ is the injection index, and $N_{0}$ is the number density of electrons per energy at $\gamma=1$ (e.g., Inoue \& Takahara 1996). We assume the minimum Lorentz factor of electrons, $\gamma_{\min }$, is equal to unity unless otherwise stated (see below). The parameter $\gamma_{\mathrm{brk}}$ is the characteristic energy above which the electron spectrum breaks by one power in the index, and is determined by the balance between radiative cooling and advective escape and/or adiabatic energy loss,

$\gamma_{\mathrm{brk}}=\frac{3 m_{\mathrm{e}} c^{2}}{4\left(u_{B}+u_{\mathrm{soft}}\right) \sigma_{\mathrm{T}} R} \beta_{\mathrm{sh}}$, where $u_{B}$ is the magnetic energy density, $u_{\text {soft }}$ is the seed photon energy density, $R$ is the source radius and $\beta_{\text {sh }}$ is the shock velocity normalized to the velocity of light.

The parameter $\gamma_{\max }$ could be determined by equating the radiative cooling time with the acceleration time scale. Although the acceleration time is not as well understood as the cooling time (via the synchrotron and inverse-Compton losses), it can be approximated by considering the mean free path, $\lambda(\gamma)$, for the scattering of electrons with magnetic disturbances. Taking the mean free path to be proportional to the Larmor radius, $R_{\mathrm{g}}$, by introducing another parameter, $\xi\left[=\lambda / R_{\mathrm{g}}\right]$, the maximum energy of electrons is given by

$\gamma_{\max }=\left[\frac{9 e B}{80\left(u_{B}+u_{\mathrm{soft}}\right) \sigma_{\mathrm{T}} \xi}\right]^{1 / 2} \beta_{\mathrm{sh}}$.

We note that the break in the electron population, of course, will not appear if $\gamma_{\text {brk }}$ is larger than $\gamma_{\max }$. In this case, Eq. (1) will be reduced to a simpler form

$N(\gamma)=N_{0} \gamma^{-\mathrm{s}} \exp \left(-\frac{\gamma}{\gamma_{\max }}\right)$ 
Table 4. Comparison of fitting parameters of the SED for knot C.

\begin{tabular}{|c|c|c|c|c|}
\hline Parameters & $\overline{(\mathrm{II}) \mathrm{Sy}(\mathrm{WCL})^{a}}$ & $\overline{(\mathrm{II}) \mathrm{Sy}(\mathrm{MCL})^{b}}$ & $\overline{(\mathrm{III}) \mathrm{Sy}+\mathrm{SSC}(\mathrm{WCL})^{c}}$ & (IV)Sy + SSC(MCL) \\
\hline$R(\mathrm{pc})$ & 250 & 250 & 250 & 250 \\
\hline$B(\mu \mathrm{G})$ & 570 & 240 & 12 & 8 \\
\hline$N_{0}\left(\mathrm{~cm}^{-3}\right)^{c}$ & $1.3 \times 10^{-2}$ & $5.8 \times 10^{-4}$ & 20.0 & $1.4 \times 10^{-1}$ \\
\hline$s^{e}$ & 2.8 & 2.2 & 2.8 & 2.2 \\
\hline$\gamma_{\min }$ & 1 & 1 & 1 & 1 \\
\hline$\gamma_{\text {brk }}$ & - & $2.6 \times 10^{4}$ & - & $1.5 \times 10^{5}$ \\
\hline$\gamma_{\max }$ & $6.0 \times 10^{6}$ & $2.4 \times 10^{7}$ & $1.4 \times 10^{7}$ & $4.0 \times 10^{7}$ \\
\hline$u_{\mathrm{e}} / u_{B}$ & 1.0 & 1.0 & $3.6 \times 10^{6}$ & $2.3 \times 10^{5}$ \\
\hline$u_{\text {sync }}\left(\mathrm{erg} \mathrm{cm}^{-3}\right)$ & $1.2 \times 10^{-11}$ & $1.4 \times 10^{-11}$ & $9.7 \times 10^{-12}$ & $1.4 \times 10^{-11}$ \\
\hline$L_{\mathrm{kin}}\left(\mathrm{erg} \mathrm{s}^{-1}\right)^{e}$ & $1.5 \times 10^{45} \Gamma_{\mathrm{BLK}}^{2}$ & $2.6 \times 10^{44} \Gamma_{\mathrm{BLK}}^{2}$ & $1.1 \times 10^{48} \Gamma_{\mathrm{BLK}}^{2}$ & $3.2 \times 10^{46} \Gamma_{\mathrm{BLK}}^{2}$ \\
\hline$L_{\mathrm{E}}\left(\mathrm{erg} \mathrm{s}^{-1}\right)^{f}$ & $1.9 \times 10^{45} \Gamma_{\mathrm{BLK}}^{2}$ & $3.4 \times 10^{44} \Gamma_{\mathrm{BLK}}^{2}$ & $1.5 \times 10^{48} \Gamma_{\mathrm{BLK}}^{2}$ & $4.3 \times 10^{46} \Gamma_{\mathrm{BLK}}^{2}$ \\
\hline$\beta_{\mathrm{sh}}^{g}$ & - & $5 \times 10^{-2}$ & - & $2 \times 10^{-3}$ \\
\hline$\xi^{\mathrm{h}}$ & $9.9 \times 10^{4} \beta_{\mathrm{sh}}^{2}$ & 36.8 & $3.2 \times 10^{5} \beta_{\mathrm{sh}}^{2}$ & 0.1 \\
\hline$t_{\text {cool }}(\mathrm{s})^{i}$ & $3.9 \times 10^{8} \gamma_{\max } \gamma^{-1}$ & $5.6 \times 10^{8} \gamma_{\max } \gamma^{-1}$ & $1.4 \times 10^{11} \gamma_{\max } \gamma^{-1}$ & $4.8 \times 10^{10} \gamma_{\max } \gamma^{-1}$ \\
\hline$c t_{\text {cool }}(\mathrm{pc})^{j}$ & $3.8 \gamma_{\max } \gamma^{-1}$ & $5.4 \gamma_{\max } \gamma^{-1}$ & $1400 \gamma_{\max } \gamma^{-1}$ & $465 \gamma_{\max } \gamma^{-1}$ \\
\hline CL-brk? & $x$ & 0 & 0 & $\mathrm{O}$ \\
\hline$\xi \geq 1 ?$ & $\bigcirc$ & 0 & 0 & $x$ \\
\hline
\end{tabular}

${ }^{a}$ Corresponding figure is Fig. 7 (I).

${ }^{b}$ Corresponding figure is Fig. 7 (II).

${ }^{c}$ Corresponding figure is Fig. 7 (III).

${ }^{d}$ Corresponding figure is Fig. 7 (IV).

${ }^{e}$ Kinetic power of electrons in knot C. $\Gamma_{\mathrm{BLK}}$ is the bulk Lorentz factor of knot C.

${ }^{f}$ Total power of electrons in knot $\mathrm{C}$.

${ }^{g}$ Shock velocity of knot $\mathrm{C}$.

${ }^{h}$ The acceleration parameter given in the text, where $\beta_{\text {sh }}$ is the shock velocity in the jet.

${ }^{i}$ Cooling time of electrons of energy $\gamma m_{\mathrm{e}} c^{2}$.

${ }^{j}$ The distance travelled by an electron before losing its energy by radiation.

Following Kino \& Takahara (2003), we call the latter situation "weak cooling (WCL)" since radiative cooling is not efficient, and the injected electron spectrum keeps its original form. Meanwhile, "moderate cooling (MCL)" represents the former situation $\left(\gamma_{\mathrm{brk}}<\gamma_{\max }\right)$, producing a cooling break in the electron spectral distribution (Eq. (1)).

Our goal is to derive the above jet parameters so as not to conflict with the observational results. Assuming a spherical geometry for the emission region, the radius of the knot $\mathrm{C}$ in 3C 15 can be set to $R=7.7 \times 10^{20} \mathrm{~cm}$ (corresponding to the FWHM of $0.5 \mathrm{kpc}$; see Sect. 2.2). We do not consider Doppler beaming/de-beaming effects and take $\delta \simeq 1$, following the suggestion of Leahy et al. (1997). The injection index of electrons can be determined either by the radio-optical index $\alpha_{\text {ro }}$ or optical-X-ray index $\alpha_{\text {ox }}: s=1+2 \alpha_{\text {ro }}=2.8$ for WCL, whereas $s=2 \alpha_{\text {ox }}=2.2$ for MCL. We can estimate the synchrotron luminosity integrated over all frequencies using the formula given by Band \& Grindlay (1985), with the result that $L_{\text {sync }} \simeq$ $10^{42} \mathrm{erg} \mathrm{s}^{-1}$. Thus the synchrotron photon energy density is $u_{\text {sync }} \simeq 10^{-11} \mathrm{erg} \mathrm{cm}^{-3}$ (see also Table 4). Comparing this with the cosmic microwave background (CMB) photon energy density boosted in the jet, $u_{\mathrm{CMB}}=4.1 \times 10^{-13}(1+z)^{4} \Gamma_{\mathrm{BLK}}^{2}=$ $5.4 \times 10^{-13} \Gamma_{\mathrm{BLK}}^{2}$ erg $\mathrm{cm}^{-3}$, the synchrotron photon energy density is more than a factor of three larger. Here the $\Gamma_{\mathrm{BLK}}$ is bulk Lorentz factor of the jet and we assume $\Gamma_{\mathrm{BLK}} \simeq 2.3$ (corresponding to $\beta=0.9$ and $\theta=45^{\circ}$; see Sect. 2.1). This indicates that the dominant source of seed photons which are upscattered is synchrotron photons, $u_{\text {soft }} \simeq u_{\text {sync }}$ (synchrotron self-Compton (SSC) dominated).

In the following, we consider four possible cases to account for overall SEDs:

(I) Synchrotron X-ray emission under the weak cooling [Sy, WCL];

(II) as for (I), but with moderate cooling [Sy, MCL];

(III) A composite synchrotron plus SSC X-ray emission under the weak cooling [Sync+SSC, WCL];

(IV) as for (III), but with moderate cooling [Sync+SSC, MCL].

\section{(I) Synchrotoron, weak cooling}

We first consider a scenario in which the X-ray emission is purely due to synchrotron emission from a power-law electron distribution (Eq. (4)). In this case, we cannot determine physical quantities uniquely, since the observed SED can be reproduced by any choice of magnetic field $u_{B}$ or electron energy density $u_{\mathrm{e}}$ such that $L_{\mathrm{sync}} \propto u_{\mathrm{e}} u_{B}$. Here we assume the conventional equipartition condition between particles and fields $\left(u_{\mathrm{e}}=\right.$ $u_{B}$ ) for simplicity. Figure 7 (I) shows the SED fit assuming the parameters listed in Table 4 . We found that a magnetic field of $B_{\text {eq }}=570 \mu \mathrm{G}$ is required to reproduce the spectra in equipartition, which corresponds to $u_{\mathrm{e}}=u_{B}=1.3 \times 10^{-8} \mathrm{erg} \mathrm{cm}^{-3}$. 
Comparing this with the synchrotron photon energy density, $u_{\mathrm{sync}}=1.2 \times 10^{-11} \mathrm{erg} \mathrm{cm}^{-3}$, both the field and particle energy densities are about 1000 times larger than the radiation energy density.

Since the peak frequency of synchrotron emission of an electron of energy $\gamma m_{\mathrm{e}} c^{2}$ is given by $v \simeq 10^{6} B \gamma^{2}(1+z)^{-1}$, $\gamma_{\max } \simeq 6.0 \times 10^{6}$ is needed to produce $\mathrm{X}$-ray photons. The cooling time of the highest energy electrons is $3.9 \times 10^{8} \mathrm{~s}$, which corresponds to a travel distance of $c t_{\mathrm{cool}}=3.8 \mathrm{pc}$, which is much smaller than the region size of $R=250 \mathrm{pc}$. In such a situation, high energy electrons should lose their energy by radiation, and hence produce a break in the power-law index at $\gamma_{\text {brk }} \sim 0.01 \gamma_{\max } \beta_{\text {sh }}$. This is inconsistent with our original assumption of "weak cooling", $\gamma_{\mathrm{brk}} \geq \gamma_{\max }$. There are two different ways of solving this problem. We may introduce a cooling break, as is discussed in detail in (II), or alternatively, the equipartition condition $\left(u_{\mathrm{e}}=u_{B}\right)$ may not be met in this source. The latter assumption requires smaller values of $B$, since the highest energy electrons must have a longer life time. For example, in order for $\gamma_{\text {brk }} \geq 10^{7}$, the magnetic field must be $B \leq 55 \mu \mathrm{G}$ (see Eq. (2)), which is an order of magnitude smaller than the equipartition value. This latter situation is discussed in detail in (III).

\section{(II) Synchrotron, moderate cooling}

As we have discussed in (I), equipartition between the electron energy density and magnetic field energy density inevitably requires a cooling break since the magnetic field is so strong. Here we assume that both the optical and X-ray photons are produced by cooled part of the electron population $\left(\propto \gamma^{-s-1}\right)$. By setting $s=2.2$ for the electron spectral index, we obtain $\alpha_{\mathrm{R}} \simeq 0.6$ and $\alpha_{\mathrm{ox}}=1.1$, respectively. Note that the $\alpha_{\mathrm{R}}$ falls in the typical spectral index of FR-I/FR-II jets in GHz band (0.5-0.7; e.g., Bridle \& Perley 1984). Extrapolation of these two lines locates the break frequency at $v_{\text {brk }} \sim 10^{12} \mathrm{~Hz} \simeq$ $10^{6} B \gamma_{\text {brk }}^{2}$. Under these conditions, physical parameters are determined to reproduce the SED (Fig. 7 (II)). A cooling break causes two important effects on the estimation of jet quantities; (i) substantially reducing electron energy/number density below $\gamma_{\text {brk }}$, and hence (ii) reducing the equipartition magnetic field considerably $\left(B_{\mathrm{eq}}=240 \mu \mathrm{G}\right)$. We found that all the jet parameters can be determined self-consistently if the shock velocity is sub-relativistic; $\beta_{\mathrm{sh}} \simeq 0.05$. The kinetic power, the total power and the pressure of the jet are estimated by the following relations:

$L_{\mathrm{kin}} \simeq \pi R^{2} c \Gamma_{\mathrm{BLK}}^{2}\left(u_{\mathrm{e}}+u_{B}\right)$,

$L_{\mathrm{E}} \simeq \pi R^{2} c \Gamma_{\mathrm{BLK}}^{2}\left(u_{\mathrm{e}}+u_{B}+P\right)$, where

$P=\frac{1}{3}\left(u_{\mathrm{e}}+u_{B}\right)$.

We obtain $L_{\text {kin }} \simeq 2.6 \times 10^{44} \Gamma_{\mathrm{BLK}}^{2} \mathrm{erg} \mathrm{s}^{-1}, L_{\mathrm{E}}=3.4 \times$ $10^{44} \Gamma_{\mathrm{BLK}}^{2} \mathrm{erg} \mathrm{s}^{-1}$, and $P=1.5 \times 10^{-9} \mathrm{erg} \mathrm{cm}^{-3}$, respectively. We will compare these jet powers to the energy contained in the radio lobes in Sect. 4.4.

An interesting prediction from this model is that the spatial extent of knot $\mathrm{C}$ would be different at different energy bands.
This is because the radio photons are emitted by the non-cooled part of the electron population $\left(\propto \gamma^{-s}\right)$, whereas both the optical and X-ray photons are radiated by cooled electrons $\left(\propto \gamma^{-s-1}\right)$. If the emission region's size is approximately related by $R \simeq$ $c t_{\text {cool }}$ for cooled electrons, a simple relation can be expected for the region sizes; $R_{\mathrm{X}}<R_{\mathrm{opt}}<R_{\mathrm{rad}}$. Unfortunately, the image resolutions of radio $\left(0.34^{\prime \prime}\right)$, optical $\left(0.1^{\prime \prime}\right)$, and X-rays $\left(0.5^{\prime \prime}\right)$ are not sufficient to test this hypothesis, but such an approach will provide an interesting opportunity for probing the particle acceleration/deceleration mechanism in the jet in the near future.

\section{(III) Synchrotron + SSC, weak cooling}

As we have seen in Sect. 3.2, the X-ray spectrum of knot C shows weak evidence of mixed power-law (broken power-law) emission. One possibility to account for the apparent concave feature in the X-ray spectrum is the overlap of the synchrotron and SSC components in the Chandra X-ray energy band. Under this assumption, we first discuss "weak cooling" with an electron spectral index of $s=2.8$. In principal, we can constrain the physical quantities tightly by comparing the synchrotron and SSC luminosity ratio; $L_{\mathrm{sync}} \propto u_{\mathrm{e}} u_{B}$, and $L_{\mathrm{SSC}} \propto$ $u_{\mathrm{e}} u_{\text {sync }}$. In order to produce comparable synchrotron and inverse Compton luminosities, a large departure from an equipartition is unavoidable since $u_{B} \sim u_{\text {sync }}$. Assuming the observed value of $u_{\text {sync }}\left(\sim 10^{-11} \mathrm{erg} \mathrm{cm}^{-3}\right)$, we require a magnetic field of an order $B \sim 10 \mu \mathrm{G}$. In order to explain the observed synchrotron spectrum with such a weak magnetic field, the electron energy density $u_{\mathrm{e}}$ must be large, since $u_{\mathrm{e}} \propto L_{\mathrm{sync}} / u_{B}$. A more accurate calculation provides a satisfactory fit to the SED for $B \simeq 12 \mu \mathrm{G}\left(u_{B}=5.7 \times 10^{-12} \mathrm{erg} \mathrm{cm}^{-3}\right)$ and $u_{\mathrm{e}}=2.1 \times$ $10^{-5} \mathrm{erg} \mathrm{cm}^{-3}$, respectively (Fig. 7 (III)).

Note, however, that the SSC luminosity is quite uncertain because we have to infer the overall SSC spectra from the "bottom edge" of the hard X-ray spectrum. We thus consider whether or not other choices of magnetic field could also reproduce the data. For a given synchrotron luminosity $\left(L_{\mathrm{sync}}\right)$ in a given region size $(R)$, the SSC peak frequency and luminosity vary as $v_{\mathrm{ssc}} \propto B^{-1}$ and $L_{\mathrm{ssc}} \propto B^{-2}$, respectively. Therefore, variations in $B$ shift the position of the SSC peak as $L_{\mathrm{ssc}} \propto v_{\mathrm{ssc}}^{2}$. In order to achieve comparable synchrotron/SSC fluxes in the Chandra X-ray band, only a magnetic field of $B \simeq 10 \mu \mathrm{G}$ is acceptable. In such a situation, the cooling time of the highest energy electrons $\left(\gamma_{\max }=1.4 \times 10^{7}\right)$ is $1.4 \times 10^{11} \mathrm{~s}$, which corresponds to a travel distance of $1400 \mathrm{pc}$. This suggests that $\gamma_{\text {brk }}$ does not appear as long as $\beta_{\mathrm{sh}} \geq 0.2$. In contrast to the case (I), this is consistent with our original assumption of weak cooling.

While this model (III) reproduces the observed concave feature of X-ray spectrum, there are two major difficulties in understanding the derived jet quantities. First, the departure from equipartition, $u_{\mathrm{e}} / u_{B} \simeq 3.6 \times 10^{6}$, seems to be too large. One possibility to reduce the discrepancy is to adopt a larger value of $\gamma_{\min }(\gg 1)$, since the electron energy density behaves $\propto \gamma_{\min }^{-s+2}$. For example, if we assume $\gamma_{\min } \simeq 10^{3}$, then $u_{\mathrm{e}} / u_{B}$ would be $\sim 1.4 \times 10^{4}$. This is still far from equipartition, though the difference is significantly reduced. (Note, however, that such a large value of $\gamma_{\min }$ is unlikely from the viewpoint of 
shock dynamics in jets, where $\gamma_{\min } \sim \Gamma_{\mathrm{BLK}}$; Kino \& Takahara 2003.) Therefore, a large departure from equipartition cannot be avoided within the framework of model (III). The second problem is related with unreasonably large jet power expected with this model. The total power of the jet is given by $L_{\mathrm{E}}=$ $1.5 \times 10^{48} \Gamma_{\mathrm{BLK}}^{2} \mathrm{erg} \mathrm{s}^{-1}$. This jet power exceeds the total output of most bright quasars (e.g., Celotti et al. 1997), though 3C 15 is a relatively weak radio source (intermediate between FR-I and FR-II; Leahy et al. 1997).

Also if the jet X-ray emission were of inverse Compton origin, we would expect it to follow the synchrotron emission more closely (emission from low-energy electrons not affected by radiative loss), whereas in fact it is closer to the optical, suggesting that X-ray emissions are dominated by synchrotron emission. Therefore, we consider model (III) to be less plausible than (II), given the very weak evidence from the X-ray spectrum. In Sect. 4.4, we will discuss this model from the view point of the energetic link between the jet and lobes. Future observations at the radio-IR bands $\left(10^{11-13} \mathrm{~Hz}\right)$, as well as higher quality X-ray data are strongly encouraged to test the predictions of model (III).

\section{$\underline{\text { (IV) Synchtotron + SSC, moderate cooling }}$}

Finally we consider the case in which the electron distribution has a cooling break and most of the X-ray photons are produced via SSC. Following case (II), we assume $s=2.2$ and the break frequency of $v_{\mathrm{brk}} \sim 10^{12} \mathrm{~Hz}$. Figure 7 (IV) shows the SED fit using this model. The existence of a cooling break substantially reduces the electron energy density, $u_{\mathrm{e}}$, and hence reduces the discrepancy between $u_{\mathrm{e}}$ and $u_{B}$. However, the magnetic field strength is still 30 times below the equipartition value of $B_{\text {eq }}=$ $240 \mu \mathrm{G}$. The total power of the jet is estimated to be $L_{\mathrm{E}}=4.3 \times$ $10^{46} \Gamma_{\mathrm{BLK}}^{2} \mathrm{erg} \mathrm{s}^{-1}$, which is reduced by a factor of 40 from model (III). In such a situation, the shock velocity must be nonrelativistic; $\beta_{\mathrm{sh}} \simeq 2 \times 10^{-3}$.

Although this model (IV) seems to reproduce the overall SED well, it requires an acceleration parameter of smaller than unity $(\xi=0.1)$. In the conventional picture of resonant pitch angle scattering (Blandford \& Eichler 1987), $\xi$ can be identified with the ratio of energy in the non-turbulent magnetic field to that in the turbulent field. Thus, $\xi$ is expected to be larger than 1 by definition. For example, Inoue \& Takahara (1996) argued that $\xi \gg 1$ for sub-pc-scale jets in blazar type AGNs, whereas in normal plasmas such as that in the interstellar medium or supernova remnants, $\xi$ is inferred to be of order unity (e.g., Bamba et al. 2003). We thus consider $\xi<1$ is unreasonable in the framework of diffusive shock acceleration theory.

\section{Summary of Model Fitting for Knot C}

In summary, we have considered four possible models to reproduce the overall SED of knot $\mathrm{C}$ in $3 \mathrm{C} 15$. The major results of our considerations are:

(i) The synchrotron X-ray model under equipartition inevitably requires a cooling break, since high energy electrons lose their energy on very short time scales $\left(t_{\text {cool }} \leq R / c\right)$.

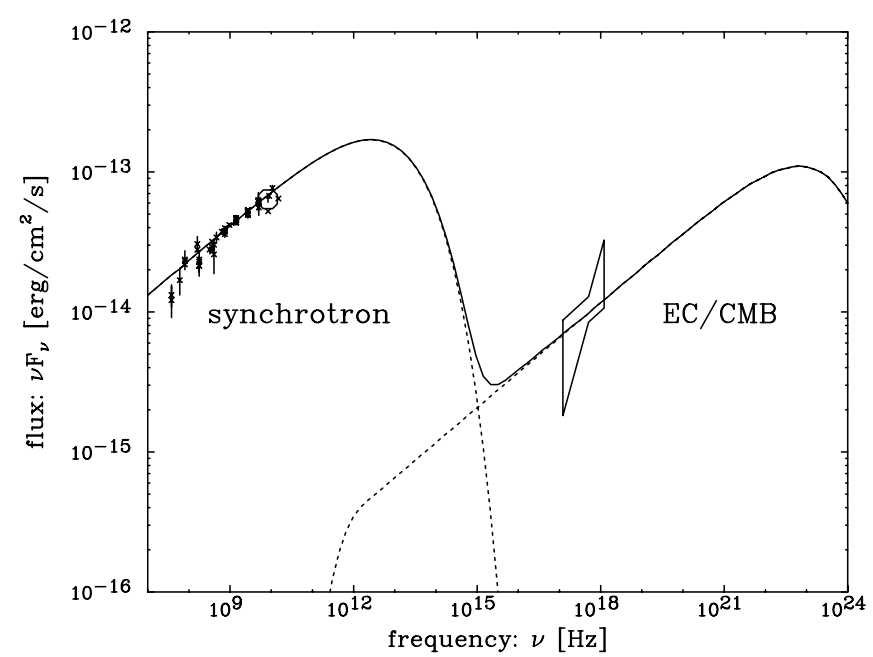

Fig. 8. SED of radio lobes in 3C 15. Bow tie: X-ray power-law spectrum obtained with Chandra (this work), Open circle: obtained by direct integration from the $3.6 \mathrm{~cm}$ radio image in Leahy et al. (1997). Crosses: NED data base, but multiplied by 0.76 .

(ii) A composite synchrotron plus SSC model predicts a magnetic field far below equipartition $\left(B_{\text {eq }} \gtrsim 30 B\right)$.

Taking these into account, we consider case (II) to be the most likely for the overall SED of knot C (Sy, MCL). Although case (III) is still possible, the large departure from equipartition, as well as the extremely large jet power, $L_{\mathrm{E}}>10^{48} \mathrm{erg} \mathrm{s}^{-1}$, seems to be problematic. In all four models, (I)-(IV), electrons must be accelerated up to $\gamma_{\max } \sim 10^{7}$ in knot $\mathrm{C}$ of $3 \mathrm{C} 15$, which corresponds to a travel distance of $c t_{\text {cool }} \leq 1.4 \mathrm{kpc}$ (see Table 4). Comparing this with the projected distance of knot $\mathrm{C}$ from the nucleus, $5.1 \mathrm{kpc}$, re-acceleration is clearly necessary in knot $\mathrm{C}$.

\subsection{Emission mechanism of the lobes}

In our $30 \mathrm{ksec}$ Chandra observation of 3C 15, we have detected diffuse X-ray emission closely associated with the radio lobes. The X-ray spectrum can be reproduced by a two component model consisting of soft and hard components. Considering the temperature and the luminosity of the Raymond-Smith fit, the soft X-ray component may naturally be attributed to thermal emission from the hot halo of the host galaxy (e.g., Matsushita et al. 2000). In fact, the X-ray luminosity of thermal component, $L_{\mathrm{RS}}=4.5 \times 10^{40} \mathrm{erg} \mathrm{s}^{-1}$, is well within the typical range of elliptical galaxies, $10^{39-42} \mathrm{erg} \mathrm{s}^{-1}$ (e.g., Eskridge et al. 1995).

The power-law modeling of the hard component gives a spectral index $\alpha_{\mathrm{X}}=0.33_{-0.43}^{+0.35}$. Unfortunately we cannot compare it with the synchrotron radio index of $3 \mathrm{C} 15$, since we have the radio-lobe flux at only one frequency (i.e. the $3.6 \mathrm{~cm}$ map). However, it is known that the integrated radio spectrum has $\alpha_{\mathrm{R}}=0.75$ from the NED photometry data base (http://nedwww.ipac.caltech.edu/). Since the total radio flux is almost entirely dominated by the lobes at radio frequencies (e.g., $76 \%$ of the radio flux is due to lobe emission at $3.6 \mathrm{~cm}$ ), it could be a reasonable approximation of synchrotron radio emission from radio lobes. Figure 8 shows the SED of radio lobes thus produced. The NED data, 
Table 5. Fitting parameters of the SED for radio lobes.

\begin{tabular}{ll}
\hline \hline Parameters & Sy + EC/CMB \\
\hline$V\left(\mathrm{~cm}^{3}\right)^{a}$ & $5.8 \times 10^{68}$ \\
$B(\mu \mathrm{G})$ & 3.7 \\
$N_{0}\left(\mathrm{~cm}^{-3}\right)$ & $7.0 \times 10^{-4}$ \\
$s$ & 2.5 \\
$\gamma_{\min }$ & 1 \\
$\gamma_{\max }$ & $1.0 \times 10^{6}$ \\
$u_{\mathrm{e}} / u_{B}$ & $2.1 \times 10^{3}$ \\
$u_{\text {sync }}\left(\mathrm{erg} \mathrm{cm}{ }^{-3}\right)$ & $3.5 \times 10^{-14}$ \\
$P_{\text {lobe,non-th }}\left(\mathrm{erg} \mathrm{cm}^{-3}\right)^{b}$ & $3.8 \times 10^{-10}$ \\
$E_{\text {lobe }}^{c}(\mathrm{ergs})$ & $8.9 \times 10^{59}$ \\
$t_{\text {cool }}(\mathrm{s})$ & $5.3 \times 10^{13} \gamma_{\max } \gamma^{-1}$ \\
$c t_{\text {cool }}(\mathrm{pc})$ & $5.2 \times 10^{5} \gamma_{\max } \gamma^{-1}$ \\
\hline${ }^{a}$ Volume of the radio lobes. & \\
${ }^{b}$ Pressure from non-thermal electrons in the lobes. & \\
${ }^{c}$ Total energy supplied in the radio lobes. & \\
\end{tabular}

multiplied by a factor 0.76 , are nicely consistent with the lobe flux estimated at $3.6 \mathrm{~cm}$. We can clearly see that the radio flux does not connect smoothly with the flat X-ray spectrum. We thus consider that the diffuse hard X-rays are produced via inverse Compton scattering on the electrons which emit the synchrotron photons in the radio band. The slightly different spectral index between the radio and X-rays may imply that X-ray photons are produced by lower energy electrons than those which produce the observed radio emission. In fact, Kellermann et al. (1969) reported that the spectral index does flatten somewhat at lower frequencies.

In order to determine the origin of the seed photons which are upscattered to X-ray energies, we approximate the geometry of the radio lobes as a cylinder $62.5 \mathrm{kpc}\left(50^{\prime \prime}\right)$ in length and $20 \mathrm{kpc}\left(16^{\prime \prime}\right)$ in diameter. This yields a lobe volume of $V=5.8 \times 10^{68} \mathrm{~cm}^{3}$ and a synchrotron photon energy density of $u_{\text {sync }} \sim 3.5 \times 10^{-14} \mathrm{erg} \mathrm{cm}^{-3}$ (see Table 5). Similarly, IR/optical photons from the host galaxy halo are expected to provide an energy density of $2 \times 10^{-13}(10 \mathrm{kpc} / d)^{2} \mathrm{erg} \mathrm{cm}^{-3}$, where $d$ is the distance from the nucleus to the X-ray emitting region in the lobe (Sandage 1973). Since the energy density of these seed photons falls below that of the CMB, $u_{\mathrm{CMB}}=5.4 \times$ $10^{-13} \mathrm{erg} \mathrm{cm}^{-3}$, we will consider CMB photons as the dominant seed photons for inverse Compton X-ray emission. Note, however, that IR/optical emission may dominate over the CMB in the innermost regions of the radio lobe, $d \leq 5 \mathrm{kpc}$. In this case, it may be that inverse Compton scattering of different seed photons is occurring in the X-ray (CMB seed photons) and $\gamma$-ray (IR/optical seed photons) bands.

The ratio of the radio (synchrotron) flux to the X-ray (inverse Compton) flux is therefore related by

$\frac{f_{\mathrm{X}}}{f_{\text {radio }}} \simeq \frac{u_{\mathrm{CMB}}}{u_{B}}$.

Here, we must compare the radio/X-ray fluxes which correspond to the same population of relativistic electrons.
The Lorentz factors of electrons which upscatter CMB photons $\left(v_{\mathrm{CMB}} \simeq 10^{11} \mathrm{~Hz}\right)$ to the $\mathrm{X}$-ray band $\left(v_{\mathrm{X}} \simeq 10^{17-18} \mathrm{~Hz}\right)$ would be $\gamma \simeq 10^{2.9-3.4}$. These electrons emit synchrotron photons in the frequency range of $v \simeq 10^{6-7}(B / 1 \mu \mathrm{G}) \mathrm{Hz}$, for which there is no observational data (Fig. 8). We thus extrapolate the observed radio flux by assuming the radio spectral index of $\alpha_{\mathrm{R}} \simeq$ 0.75 . We obtain $f_{\text {radio }}=2.1 \times 10^{-14}(B / 1 \mu \mathrm{G})^{1-\alpha_{\mathrm{R}}} \mathrm{erg} \mathrm{cm}^{-2} \mathrm{~s}^{-1}$ for the frequency range of $v \simeq 10^{6-7}(B / 1 \mu \mathrm{G}) \mathrm{Hz}$. Comparing this with the integrated X-ray flux of $f_{\mathrm{X}}=(2.3 \pm 0.5) \times$ $10^{-14} \mathrm{erg} \mathrm{cm}^{-2} \mathrm{~s}^{-1}$ in the Chandra bandpass, the magnetic field strength is estimated to be $B \simeq 4 \mu \mathrm{G}$. The result of SED model fitting is shown as solid lines in Fig. 8. We have assumed the maximum Lorentz factor of electrons to be $\gamma_{\max } \simeq 10^{6}$. The best fit parameters are summarized in Table 5 .

A comparison of the synchrotron and inverse Compton flux densities allows us to derive $u_{\mathrm{e}}=1.1 \times 10^{-9} \mathrm{erg} \mathrm{cm}^{-3} \simeq$ $2100 u_{B}$. Thus we find that there is a significant electron dominance in the lobes of $3 \mathrm{C} 15$. This ratio is larger than those reported for lobes in other radio galaxies $\left(u_{\mathrm{e}} / u_{B} \leq 100\right.$; e.g., Tashiro et al. 1998; Isobe 2002), but the difference is mainly due to the different choice of $\gamma_{\min }$ : we have assumed $\gamma_{\min }=1$ throughout this paper as discussed in Sect. 4.1. If we set $\gamma_{\min } \simeq$ 1000 as for these published works, we obtain $u_{\mathrm{e}} \simeq 66 u_{B}$, which is well in the range of published works. The total power supplied in the lobes is estimated as:

$E_{\text {lobe }}=\left(u_{\mathrm{e}}+u_{B}\right) V+P V \simeq 8.9 \times 10^{59} \mathrm{ergs}$.

It is interesting to compare the thermal pressure estimated from the Raymond-Smith (RS) X-ray emission, and non-thermal pressures of relativistic electrons in the radio lobe. If we assume a very simplified model in which thermal photons are produced by electrons which have a constant number density, $n_{\mathrm{e}, \mathrm{th}}$ over the radio lobes, the best fit X-ray spectral model (see 
Sect. 3.3 and Table 2) provides the number density of thermal electrons as

$n_{\mathrm{e}, \mathrm{th}}=3.1 \times 10^{-3} \mathrm{~cm}^{-3}$,

where we have assumed that the thermal plasma volume is equal to that of radio lobes; $V=5.8 \times 10^{68} \mathrm{~cm}^{3}$. The pressure of thermal plasma is thus given by

$P_{\text {lobe,th }}=1.9 n_{\mathrm{e}, \mathrm{th}} k T=8.1 \times 10^{-12} \mathrm{erg} \mathrm{cm}^{-3}$,

where $k T=0.85 \mathrm{keV}$ from Sect. 3.3 (see Table 2). Meanwhile, the non-thermal pressure of relativistic electrons is estimated as

$P_{\text {lobe,non-th }}=\frac{1}{3}\left(u_{\mathrm{e}}+u_{B}\right)=3.8 \times 10^{-10} \mathrm{erg} \mathrm{cm}^{-3}$.

The large difference between $P_{\text {lobe,non-th }}$ and $P_{\text {lobe,th }}$ may partly due to our over-simplified model for the thermal plasma distribution, but it seems that a more powerful (dense) thermal plasma, other than that emitting the X-rays, is necessary to confine the relativistic electrons in the radio lobes.

Finally, we should comment on the features of the surface brightness ratio $\left(S_{\text {radio }} / S_{\mathrm{X}}\right)$ given as Fig. $2 \mathrm{c}$. It has been claimed that this ratio represents the magnetic field distribution along the major axis of the lobes, since $S_{\text {radio }} / S_{\mathrm{X}} \propto B^{2}$ (except for the nucleus regions; see Eq. (8)). In this sense, the magnetic field may be weakest near the nucleus, increasing several-fold at larger distances, as was suggested for the field-particle distribution of the FR II radio galaxy 3C 452 (Isobe et al. 2002). However, we should note that the thermal component, if distributed over the scale of the galaxy, could be centrally condensed. These are possibly related to the thermal RS emission seen in the X-ray spectrum of the radio lobes (Fig. 5). In principle, it would be possible to discriminate between the two emission components by comparing X-ray images in different energy bands, where the soft X-rays $(\leq 2 \mathrm{keV})$ are dominated by thermal RS emission and the hard $\mathrm{X}$-rays $(\geq 2 \mathrm{keV})$ would be of non-thermal inverse Compton origin. Unfortunately, photon statistics are not sufficient for such a study. We simply comment here that the apparent center filled morphology of the $\mathrm{X}$-ray image (Fig. 2a) may partly be due to a centrally condensed thermal plasma, rather than variations in the magnetic field strength. The actual inverse-Compton profile of the radio lobes might be closer to the synchrotron radio profile than suggested in Fig. 2. Future deep X-ray observations will clarify this point further.

\subsection{Emission mechanism of the nucleus}

In Sect. 3.4, we showed that the X-ray spectrum of the nucleus consists of two different power-law components. The low-energy component is well represented by a hard power-law with Galactic absorption, whereas the high-energy component is heavily absorbed with $N_{\mathrm{H}}=10^{22-23} \mathrm{~cm}^{-2}$. One possibility to account for this X-ray spectrum is that the two components are produced in different emission regions around the nucleus: the low-energy power-law may be due to the inner-jet emission and possibly comes from the parsec-scale region, whereas the highenergy component may be emission from the hidden active nucleus at the center. Considering the symmetrical lobe morphology, the obscuring material is most likely the molecular torus fueling the nucleus which is postulated to exist in active galaxies (e.g., Antonucci \& Miller 1985; Krolik \& Begelman 1986).

Interestingly, additional evidence of a hidden active nucleus has been obtained from the surface brightness profile of $3 \mathrm{C} 15$ in the $V$ and $R$ bands. While these are very well described by a de Vaucouleurs profile, $\propto r^{1 / 4}$, between $0.5 \mathrm{kpc}$ and $10 \mathrm{kpc}$, 3C 15 lacks a sharp, point-like AGN inside a radius of $0.3^{\prime \prime}$ (Martel et al. 1998). This optical observation is consistent with the lack of strong AGN-type features in the spectrum of 3C 15 (Tadhunter et al. 1993). Two possibilities have been suggested to account for the absence of a resolved AGN, which is in contrast common to the other $3 \mathrm{C}$ radio galaxies with optical jets imaged with $H S T$. First, the central source may be presently inactive, but is activated at regular intervals, leading to the periodic ejection of material along the jet axis (resulting in knots A, $\mathrm{B}$, and C). Alternatively, heavy obscuration by dust towards the line of sight to the nucleus could also account for the nondetection of an AGN. Dust permeates the central regions of 3C 15: if the extinction is large enough, the bright nucleus would then be hidden from our line of sight as implied from the X-ray spectrum of the nucleus. It should be noted that the dust seen in the HST images is on a much larger scale than the hypothetical molecular torii around AGNs. Thus it might be possible that the dust cloud orbiting on the kpc-scale that we directly see is the material which obscures part of the X-ray core; in other words, a large "dust torus" might be present around the nucleus of 3C 15.

\subsection{The link between the jet and lobes}

Comparison of the power transported by the jet $\left(L_{\mathrm{E}}\right)$ and the total energy contained in the lobes $\left(E_{\text {lobe }}\right)$ provides an interesting opportunity to probe the dynamics in the jet. By dividing $E_{\text {lobe }}$ by $L_{\mathrm{E}}$, we can estimate the "fueling time" of the jet $t_{\text {fuel }}$, which can be compared with the source age $t_{\text {src }}$ (see Sect. 1). In model (II) of Sect. 4.1, we obtained $L_{\mathrm{E}} \simeq 3.4 \times$ $10^{44} \Gamma_{\mathrm{BLK}}^{2} \mathrm{erg} \mathrm{s}^{-1}$. The fueling time is expected to be $t_{\text {fuel }}=$ $8.2 \times 10^{7} \Gamma_{\mathrm{BLK}}^{-2}$ yrs. This is qualitatively consistent with the source age of radio lobes, $t_{\mathrm{src}} \sim 2.1 \times 10^{7}\left(0.01 \mathrm{c} / \mathrm{v}_{\mathrm{exp}}\right) \mathrm{yrs}$. In contrast, other models, in particular (III), predict significantly smaller $t_{\text {fuel }}$ compared to $t_{\text {src }}$. In fact, for $L_{\mathrm{E}} \simeq 1.5 \times$ $10^{48} \Gamma_{\mathrm{BLK}}^{2} \mathrm{erg} \mathrm{s}^{-1}$, we obtain $t_{\text {fuel }}=1.9 \times 10^{4}$ yrs.

These facts suggest again that the synchrotron emission with moderate cooling, case (II), provides a reasonable explanation of the X-ray emission mechanism of knot C. However, there are still several possibilities to account for the apparent discrepancy in model (III), i.e., $t_{\text {fuel }} \ll t_{\text {src }}$. First, we may have underestimated the total energy in the lobes since we only take account of relativistic electrons. In fact, we cannot estimate the contribution from thermal electrons and possibly protons which should constitute a reservoir for acceleration of non-thermal electrons. If only $0.1 \%$ of electrons are "visible" in the radio and X-ray bands, the discrepancy may be reduced significantly. (However, we note that the situation is the same for the total power of the jet, $L_{\mathrm{E}}$, in the sense that we only take account of the contribution from "visible" relativistic electrons.) 
Alternatively, it is plausible that by considering the brightest jet knot (knot $\mathrm{C})$ we have overestimated the jet power. The knots are thought to be where the particle and/or field densities are enhanced compared to the rest of the jet. It may be reasonable to assume that the jet is actually very sparse, and that its average power is much lower, e.g., $0.1 \%$ of that in the bright knot. It might be said that the jet has a spatial filling factor of only $\sim 10^{-3}$. Such uneven jet structure may be related with the activity of the central nucleus. If the nucleus expels blobs of material intermittently, rather than in a stationary manner, it will produce bright knots along the jet axis at semi-regular intervals (such as that observed for knots A, B, and C). This idea can be readily accommodated by the internal shock scenario, recently proposed by a number of authors to account for the variability properties of sub-pc scale jets (e.g., Spada et al. 2001; Kataoka et al. 2001; Tanihata et al. 2003). Although somewhat speculative, occasional activity of the jet ( $0.1 \%$ of the time) may explain the energy link between the jets and the lobes in radio galaxies quite well. Future observations in the hard X-ray and gamma-ray bands are necessary to test this further.

\section{Conclusion}

We have reported the X-ray detection of the jet, lobes, and absorbed nucleus of the FR II radio galaxy 3C 15 . The X-ray image obtained with Chandra clearly shows that most of the $\mathrm{X}$-ray jet emission comes from knot $\mathrm{C}$, which was previously detected in the radio and optical jets. We found that the X-ray flux is well below the extrapolation from the radioto-optical continuum. We consider four possible cases to reproduce the overall SED: (I) weak synchrotron cooling with $u_{\mathrm{e}}=u_{B}$, (II) moderate synchrotron cooling with $u_{\mathrm{e}}=u_{B}$, (III) weak synchrotron plus SSC cooling, and (IV) moderate synchrotron plus SSC cooling. Case (I) and (IV) were safely ruled out by considering the acceleration and radiative cooling processes over the source. We argue that case (II) is a reasonable scenario to understand the SED of knot $\mathrm{C}$, but the case (III) is possible if equipartition is strongly violated $\left(u_{\mathrm{e}} / u_{B} \simeq 3.6 \times 10^{6}\right)$ and the jet power is extremely large $\left(L_{\mathrm{E}} \simeq\right.$ $10^{48} \mathrm{erg} \mathrm{s}^{-1}$ ). In either case, the highest energy electrons, with $\gamma_{\max } \geq 10^{7}$, need to be re-accelerated in knot C. The diffuse hard X-ray emission associated with the lobes is most likely due to the inverse Compton emission of CMB photons by the synchrotron emitting electrons in the radio lobes. Assuming a radio spectral index of $\alpha_{\mathrm{R}} \simeq 0.75$, we found that the lobes in 3C 15 are particle dominated, where $u_{\mathrm{e}} / u_{B} \sim 2100$. The fueling time $\left(t_{\text {fuel }}\right)$ is qualitatively consistent with the source age $t_{\text {src }}$ for the case (II), whereas the latter must be significantly shorter for the case (III). The discrepancy of (III), however, can be understood if the jet is actually very sparse, and has a spatial filling factor of only $10^{-3}$. By comparing the thermal pressure associated with the galaxy halo and non-thermal pressure in the radio lobe, we found that relativistic electrons cannot be confined only with the X-ray emitting thermal gas. Finally, we show that the X-ray emission from the nucleus consists of two power-law components, one of which suffers from significant absorption $N_{\mathrm{H}} \simeq 10^{22-23} \mathrm{~cm}^{-2}$ intrinsic to the source. Such a high column density may support the existence of dusty torus around the nucleus, as suggested by the optical observations.

Acknowledgements. We appreciate the insightful comments and suggestions of the referee, Dr. M. Georganopoulos, that have improved this paper. We also thank Dr. N. Isobe for useful discussion of X-ray properties of radio lobes. J.K. acknowledges a support by JSPS.KAKENHI (14340061). This research has made use of the NASA/IPAC Extragalactic Database (NED) which is operated by the Jet Propulsion Laboratory, California Institute of Technology, under contract with the National Aeronautics and Space Administration.

\section{References}

Antonucci, R., \& Miller, J. 1985, ApJ, 297, 621

Bamba, A., Yamazaki, R., Ueno, M., \& Koyama, K. 2003, ApJ, 589, 827

Band, D. L., \& Grindlay, J. E. 1985, ApJ, 298, 128

Blandford, R. D., \& Eichler, D. 1987, Phys. Rep., 154, 1

Bridle, A. H., \& Perley, R. A. 1984, ARA\&A, 22, 319

Celotti, A., Padovani, P., Ghisellini, G., et al. 1997, MNRAS, 286, 415

Eskridge, P. B., Fabbiano, G., \& Kim, D.-W. 1995, ApJS, 97, 141

Georganopoulos, M., \& Kazanas, D. 2003, ApJ, 589, L5

Hardcastle, M. J., Birkinshaw, M., \& Worrall, D. M. 2001, MNRAS, 326, 1499

Hardcastle, M. J., Birkinshaw, M., Cameron, R. A., et al. 2002, ApJ, 581,948

Harris, D. E., \& Krawczynski, H. 2002, ApJ, 565, 244

Inoue, S., \& Takahara, F. 1996, ApJ, 463, 555

Isobe, N., Tashiro, M., Makishima, K., et al. 2002, ApJ, 580, L111

Isobe, N. 2002, Ph.D. Thesis, University of Tokyo

Kaneda, H., Tashiro, M., Ikebe, Y., et al. 1995, ApJ, 453, L13

Kataoka, J., Takahashi, T., Wagner, S. J., et al. 2001, ApJ, 560, 659

Kataoka, J., Edwards, P., Georganopoulos, M., Takahara, F., \& Wagner, S. 2003, A\&A, 399, 91

Kellermann, K. I., Pauliny-Toth, I. I, \& Williams, P. J. S. 1969, ApJ, 157,1

Kino, M., \& Takahara, F. 2003, MNRAS, submitted

Krolik, J., \& Begelman, M. 1986, ApJ, 308, L55

Leahy, J. P., Black, A. R. S., Dennett-Thorpe, J., et al. 1997, MNRAS, 291, 20

Leahy, J. P., \& Gizani, N. A. B. 2001, ApJ, 555, 709

Martel, A. R., Sparks, W. B., Macchetto, D., et al. 1998, ApJ, 496, 203

Matsushita, K., Ohashi, T., \& Makishima, K. 2000, PASJ, 52, 685

Sambruna, R. M., Urry, C. M., Tavecchio, F., et al. 2001, ApJ, 549, L161

Sandage, A. 1973, ApJS, 183, 711

Scheuer, P. A. G. 1995, MNRAS, 277, 331

Spada, M., Ghisellini, G., Lazzati, D., \& Celotti, A. 2001, MNRAS, 325,1559

Stark, A. A., Gammie, C. F., Wilson, R. W., et al. 1992, ApJS, 79, 77

Tadhunter, C. N., Morganti, R., di Serego Alighieri, S., et al. 1993, MNRAS, 263, 999

Tanihata, C., Takahashi, T., Kataoka, H., \& Madejski, G. M. 2003, ApJ, 584, 153

Tashiro, M., Kaneda, H., Makishima, K., et al. 1998, ApJ, 499, 713

Tashiro, M., Makishima, K., Iyomoto, N., Isobe, N., \& Kaneda, H. 2001, ApJ, 546, L19

Wilson, A. S., Young, A. J., \& Shopbell, P. L. 2001, ApJ, 547, 740

Wilson, A. S., \& Yang, Y. 2002, ApJ, 568, 133 